\title{
Electron beam charge diagnostics for laser plasma accelerators
}

\author{
K. Nakamura, ${ }^{1}$ A. J. Gonsalves, ${ }^{1}$ C. Lin, ${ }^{1,2}$ A. Smith, ${ }^{1}$ D. Rodgers,,${ }^{1}$ R. Donahue, ${ }^{1}$ W. Byrne,,${ }^{1}$ and W. P. Leemans ${ }^{1,3}$ \\ ${ }^{1}$ Lawrence Berkeley National Laboratory, University of California, Berkeley, California 94720, USA \\ ${ }^{2}$ Peking University, Beijing, 100871, People's Republic of China \\ ${ }^{3}$ Department of Physics, University of California, Berkeley, California 94720, USA
}

(Received 23 December 2010; published 27 June 2011)

\begin{abstract}
A comprehensive study of charge diagnostics is conducted to verify their validity for measuring electron beams produced by laser plasma accelerators (LPAs). First, a scintillating screen (Lanex) was extensively studied using subnanosecond electron beams from the Advanced Light Source booster synchrotron, at the Lawrence Berkeley National Laboratory. The Lanex was cross calibrated with an integrating current transformer (ICT) for up to the electron energy of $1.5 \mathrm{GeV}$, and the linear response of the screen was confirmed for charge density and intensity up to $160 \mathrm{pC} / \mathrm{mm}^{2}$ and $0.4 \mathrm{pC} /\left(\mathrm{ps} \mathrm{mm}^{2}\right)$, respectively. After the radio-frequency accelerator based cross calibration, a series of measurements was conducted using electron beams from an LPA. Cross calibrations were carried out using an activationbased measurement that is immune to electromagnetic pulse noise, ICT, and Lanex. The diagnostics agreed within $\pm 8 \%$, showing that they all can provide accurate charge measurements for LPAs.
\end{abstract}

DOI: 10.1103/PhysRevSTAB.14.062801

\section{INTRODUCTION}

Laser plasma accelerators (LPAs) [1] have shown remarkable progress over the past decade, driven in part by advances in laser technology. The production of quasimonoenergetic electron beams (e-beams) with energies of the order of $100 \mathrm{MeV}$ in a few millimeters was demonstrated in 2004 [2-4]. In 2006, the production of GeV electron beams was demonstrated in just a few centimeters $[5,6]$, using a discharge capillary based guiding structure [7]. Several injection schemes have been proposed to improve stability and quality of e-beams [8-10], and initial experiments have shown promising results [11-15]. This progress is making LPAs attractive as a driver for a hyperspectral light source that produces electromagnetic radiation ranging from $\mathrm{THz}[16]$ to $\mathrm{x}$ ray $[17,18]$.

A precise measurement of electron beam (e-beam) charge is essential for any kind of accelerator. Numerous technologies have been developed for conventional radiofrequency accelerators (RFAs) such as Faraday cups, integrating current transformers (ICTs) [19], and cavity based diagnostics. Since an LPA can provide e-beams with a wide range of energy spreads (from $1 \%$ level to $100 \%$ ), angular divergence, and charge, various techniques including previously mentioned technologies have been employed: surface barrier detectors [20], scintillators with photomultipliers [21], cloud chambers [20], scintillating fibers [22], activation-based measurements [23], thermoluminescent dosimeters [24], radiochromic film [25],

Published by the American Physical Society under the terms of the Creative Commons Attribution 3.0 License. Further distribution of this work must maintain attribution to the author(s) and the published article's title, journal citation, and DOI.
PACS numbers: 06.20.fb, 07.77.Ka, 29.20.-c, 29.40.Mc

imaging plates (IPs) [3], and scintillating screens with cameras $[20,21]$.

Detection by surface barrier detectors and scintillators with photomultipliers were popular methods in early LPA experiments due to their high sensitivity. Scintillating fibers added the capability of imaging, but their cost became significant for large scale systems that cover a broad energy range. Scintillating screens and IPs are now widely used as they allow large areas to be imaged with reasonable sensitivity and cost. Combined with a dipole magnet, they can provide e-beam energy spectrum information over a broad spectral range [26-28].

As an IP is capable of accumulative measurements, it can have an advantage in sensitivity. By using monoenergetic e-beams from RFAs, the signal of the IP against relativistic electrons up to $1 \mathrm{GeV}$ of electron energy was experimentally calibrated against the e-beam charge measured by a Rogowsky coil or current transformer $[29,30]$. The range of applicable charge density was extensively studied with $40 \mathrm{MeV}$ e-beams from an RFA [31] using an ICT and Faraday cup measurements for reference.

As LPAs can have significant shot-to-shot fluctuation in the electron beam properties depending on the input parameters, high repetition-rate single-shot measurements of e-beams are important. Scintillating screens are ideal for typical $10 \mathrm{~Hz}$ repetition-rate operation since the fluorescence decay time is less than $10 \mathrm{~ms}$. Among many kinds of scintillating screens from various manufactures, the ones with terbium doped gadox $\left(\mathrm{Gd}_{2} \mathrm{O}_{2} \mathrm{~S}: \mathrm{Tb}\right)$ as an active layer have been commonly used in the LPA community. The light yield from the screens has been experimentally calibrated against ICTs by using e-beams from RFA with 3-8 MeV electron energy [26] and $40 \mathrm{MeV}$ electron energy [32]. By using broadband electron beams from an LPA, sensitivity for 1 to $80 \mathrm{MeV}$ electrons was experimentally 
calibrated against IPs [28]. Although simulations suggested that the scintillating screens were energy insensitive above a few $\mathrm{MeV}$, a detailed experimental study with electrons above $80 \mathrm{MeV}$ has not been reported until now. Since recent progress in LPA research has pushed attainable energy to $1 \mathrm{GeV}$ and beyond [5,6,33,34], it is important to experimentally explore the applicable energy range of the screens at those energies.

Faraday cups and ICTs have been used as reliable charge diagnostics in the RFA community [19,31]. Since Faraday cups have to physically capture electrons, their size can be fairly large to stop $\mathrm{GeV}$ electrons. In contrast, ICTs are nondestructive, energy independent, and compact. Despite all of the favorable features of the ICT for LPA, its use for LPA produced e-beams has been questioned in recent studies. It was reported in Ref. [26] that the ICT overestimated the e-beam charge by more than an order of magnitude compared to the measurement based on the RFA-calibrated scintillating screen, and the source of discrepancy was attributed to the electromagnetic pulse (EMP) from the laser-plasma interaction. Another cross calibration using LPA produced e-beams was done in Ref. [35], where it was reported that an ICT overestimated the charge by a factor of about 3-4 compared to the IP based charge measurements. Both studies indicated that further cross calibration measurements and detailed investigations were necessary regarding the use of the ICT in a harsh laser-plasma environment.

In this paper, we have experimentally studied the sensitivity of Lanex Fast scintillating screen (Kodak, Rochester, NY, United States) using e-beams provided by a booster synchrotron at the Advanced Light Source (ALS), Lawrence Berkeley National Laboratory (LBNL). The energy of the electron beam was varied from 106 to $1522 \mathrm{MeV}$ covering the as yet unexplored energy range. In addition, the linearity of the response against different charge density and charge intensity has been extensively studied. This study provides essential information for the Lanex to be a charge diagnostic for $\mathrm{GeV}$ e-beams.

A comprehensive study of charge diagnostics for LPAs was performed using an ICT, a Lanex Fast screen (Lanex), and an activation-based measurement [23]. The activationbased measurement is intrinsically rf noise tolerant and independent of the e-beam intensity. Therefore, it can provide an accurate reference for LPA produced e-beams. This RFA based calibration of Lanex was benchmarked against the activation-based measurement using LPA produced e-beams. Also the ICT was cross calibrated against Lanex using LPA produced e-beams. The results show that the Lanex and ICT can be accurate diagnostics for an LPA.

The calibration of the Lanex with RFA produced e-beams is shown in Sec. II, the cross calibration of the ICT, Lanex, and activation-based measurement with LPA produced e-beams is described in Sec. III, and conclusions are provided in Sec. IV.

\section{LANEX CALIBRATION WITH RF ACCELERATOR}

\section{A. Experimental setup}

The light yield of the Lanex for relativistic monoenergetic electron beams was studied at the booster-to-storage ring (BTS) beam line of the ALS, LBNL. The ALS linear accelerator (linac) provided $50 \mathrm{MeV}$ e-beams with a microbunch duration of $\simeq 30 \mathrm{ps}$ in full-width half-maximum (FWHM) [36], and one or two microbunches with $8 \mathrm{~ns}$ separation determined by the $125 \mathrm{MHz}$ electron gun cathode pulser. The total charge was controlled by changing the bias voltage of the thermionic electron gun (gun bias voltage). Each microbunch contained up to $\simeq 450 \mathrm{pC}$ giving the maximum macrobunch charge around $900 \mathrm{pC}$. The bunches were produced at $0.5 \mathrm{~Hz}$ of repetition rate so that thermal loading of the screen was not significant.

The e-beam from the linac was injected into the booster and further accelerated up to $1522 \mathrm{MeV}$. The e-beam was then extracted from the booster by the extraction magnet system, a part of which is shown in Fig. 1. In normal operation, the e-beam is extracted at the top of the booster magnets' current ramp, which gave $1522 \mathrm{MeV}$, the highest e-beam energy. By changing the e-beam extraction trigger timing and field strength of the extraction magnets, an e-beam with lower energy was extracted from the booster and sent to the BTS beam line.

Inside of the BTS beam line, there was a magnet (FE-B1, see Fig. 1) to send e-beams to the Lanex calibration setup that is shown in Fig. 2, instead of to the storage ring. The FE-B1 magnet was also used to determine the energy of the e-beam using the following procedure. First, with the FE-B1 magnet off, the e-beam was aligned to the center of the beam line by using four steering magnets and two monitors that were installed before and after the FE-B1 magnet (see Fig. 1 for locations) to establish the injection angle and position offset consistently for e-beams with

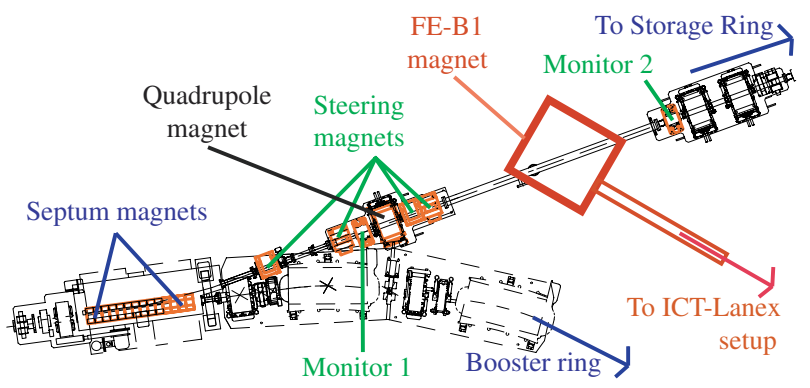

FIG. 1. Schematic of a part of the booster synchrotron and a part of the booster-to-storage beam line of the Advanced Light Source. The first pair of the steering magnets (horizontal and vertical) was used to align e-beams on monitor 1 , and second pair for monitor 2 in the same manner. Shown by the square is the location of the FE-B1 magnet that was used to send e-beams to the experimental setup shown in Fig. 2. The location of the last quadrupole magnet for this experiment is indicated as well. 


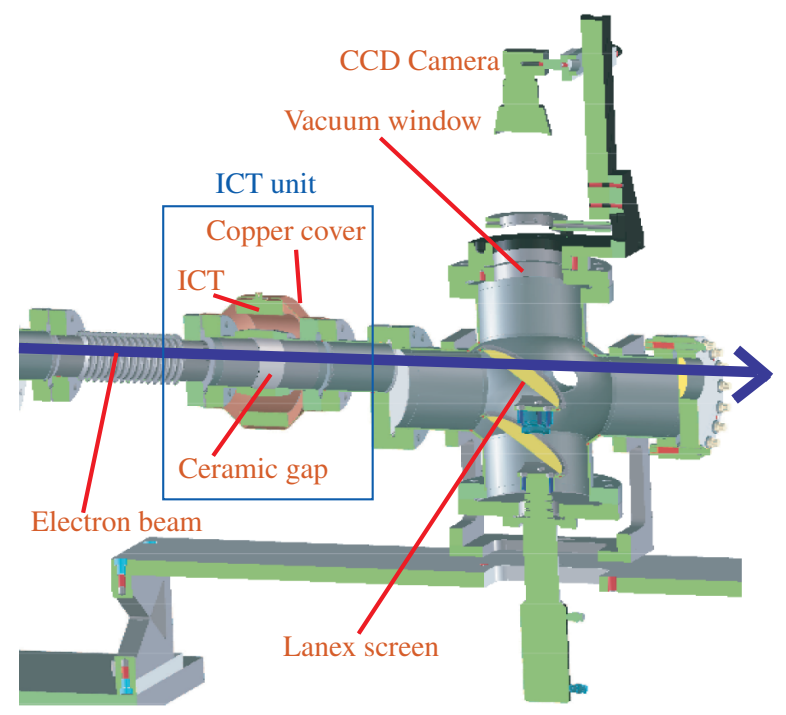

FIG. 2. Setup for the Lanex-ICT cross calibration with RFA produced e-beams. Electron beams came from the left going through an ICT (ICT-122-070-05:1) and Lanex screen (Lanex Fast Front or Back) in a vacuum tube. The ICT was installed over a ceramic gap, and a copper cover outside of the ICT provided a path for the return current. A rubber insulator was used between the ceramic gap and the ICT as well as a sheet of Kapton between the ICT and copper cover. The light emitted by the Lanex was observed by a 12-bit CCD camera (FLEA-HIBW, Point Grey), which was installed outside of the vacuum tube.

different energies. The excitation current of the FE-B1 magnet was then scanned to find the e-beam at the Lanex screen installed in the end of the beam line. Using the excitation current for $1522 \mathrm{MeV}$ e-beam as the reference, the energies of e-beams were deduced.

The longitudinal bunch duration, momentum spread, and transverse emittance of the e-beam evolved during the acceleration in the booster. Although they were not measured during the experiments described in the paper, they were modeled theoretically [37]. The longitudinal bunch duration was estimated to be $\simeq 200$ ps FWHM for $1522 \mathrm{MeV}$ e-beams. Note that the bunch duration of the injected e-beam was $\simeq 30 \mathrm{ps}$. In order to give a lower-limit estimate to the charge density and charge intensity at the Lanex screen, a bunch duration of 200 ps FWHM was assumed for all the energies explored in this paper. For the charge density estimate, e-beams with two microbunches was considered to be one bunch, because the microbunch separation time of 8 ns was much shorter than the fluorescence decay time. For the charge intensity, only e-beams with one microbunch were considered. The root-mean-square (rms) momentum spread of the e-beam was estimated to be $\simeq 0.4 \%$ to $\simeq 0.063 \%$, so hence e-beams were considered to be monoenergetic.

The incident angle of the electrons on the Lanex screen can in principle affect the light yield as well, because it defines the interaction length between the electron and the active layer of the Lanex. The last focusing quadrupole magnet was located before the second pair of the steering magnets, and this was $\sim 5 \mathrm{~m}$ away from the Lanex-ICT setup. The magnet was tuned to provide a focus at the Lanex. The largest possible convergence angle of the e-beam was estimated to be $3.2 \mathrm{mrad}$ based on the clear aperture of the quadrupole magnet, which is $32.5 \mathrm{~mm}$ in diameter. For the normalized transverse emittance, the evolution during the acceleration is estimated to be in the range between $\simeq 3 \mathrm{~mm} \mathrm{mrad}$ and $\simeq 3 \times 10^{-2} \mathrm{~mm} \mathrm{mrad}$ [37]. Based on the low transverse emittance and the small convergence angle, all electrons in a beam were considered to be parallel to the beam line throughout the paper.

Two types of Lanex Fast were studied: Lanex Fast Front (thinner) and Lanex Fast Back (thicker). Since the Lanex Fast was the most sensitive model among Lanex screens and the Lanex Fast Back was employed to an electron spectrometer developed for GeV-LPA experiments at LBNL [27], they were studied in this work. An ICT (ICT-122-070-05:1, Bergoz Instrumentation, Saint Genis Pouilly, France) was used as the reference. Shown in Fig. 2 is the setup for the Lanex-ICT calibration experiment. Electron beams passed through the ICT and Lanex in a vacuum tube. The ICT was installed over a ceramic gap to enable measurement of the e-beam current, and a copper cover outside of the ICT provided a path for the return current going through the vacuum wall. A rubber insulator was used between the ceramic gap and the ICT as well as a sheet of Kapton between the ICT and copper cover to ensure that it was well insulated.

Signals from the ICT were measured by a oscilloscope (TDS 3054B, Tektronix Inc., Beaverton, OR, United States), and the waveforms were recorded for postanalysis. The light emitted by the Lanex went through a borosilicate vacuum window, and was measured by a 12-bit charge coupled device (CCD) camera (FLEA-HIBW, Point Grey, Richmond, BC, Canada) with objectives of effective focal length $6.4 \mathrm{~mm}$ and $f$ number 1.4. Note that, although the readout of the camera was provided by a 12-bit analogdigital converter, a practical bit depth defined by the ratio of the well depth to the noise was $\simeq 10$ bit. The camera was installed outside of the vacuum tube and $213 \pm 5 \mathrm{~mm}$ away from the screen, and used in $2 \times 2$ binning mode giving 512 (horizontal) $\times 384$ (vertical) pixels throughout the experiments. The Lanex screen was installed at a 45-degree to the incident angle of the e-beam (see Fig. 2), providing an environment where the CCD camera could observe the emitted light without a mirror and minimizing noise due to the bremsstrahlung. The shape of the Lanex screen was elliptic with the horizontal diameter of $80 \mathrm{~mm}$ and the vertical diameter of $95 \mathrm{~mm}$.

The upstream surface of the Lanex was covered by a $\simeq 40 \mu \mathrm{m}$ thick aluminum foil to minimize any contributions from scattered laser light, which copropagates with the electron beam when exiting from the LPA [27]. To mimic the LPA setup, all RFA based calibration 
experiments were also carried out with the aluminum foil in front of the LANEX.

For charge measurement using the ICT, the temporal waveform of the ICT signal was recorded for each shot, a typical example of which is shown in Fig. 3. For each shot, the background level was evaluated by averaging the signal between $0 \mathrm{~ns}$ (when the external trigger was applied for the scope acquisition) and the first vertical dotted line in Fig. 3. The evaluated background level is indicated by the dashed line. To obtain charge, the signal between the second and the third vertical dotted line was integrated. The signal to noise ratio was defined by $S N_{\text {ict }}=V_{\text {peak }} / V_{\sigma \mathrm{BG}}$, where $V_{\text {peak }}$ is the peak signal voltage and $V_{\sigma \mathrm{BG}}$ is the standard deviation of the background signal.

In order to estimate the number of photons emitted by the Lanex, the following optical properties of the system were measured. The transmission of the borosilicate window was measured to be $(0.95 \pm 0.02)$, and of the objectives was $(0.88 \pm 0.02)$ for the wavelength of $543 \mathrm{~nm}$. Note that the emission peak of the Lanex is at $546 \mathrm{~nm}$. The quantum efficiency of the camera at $550 \mathrm{~nm}$ was 0.46 , and the analog to digital unit for $2 \times 2$ binning mode was 9.3 (9.3 electrons for one digital count) [38]. Based on the distance between the Lanex and the camera, the $f$ number, and the focal length, the solid angle of the system was $(3.6 \pm 0.1) \times 10^{-4}$ sr. Assuming the Lambertian distribution for the directionality of the emitted light [26,32], the light collection efficiency of the system was $(1.2 \pm 0.1) \times$ $10^{-4}$. The total efficiency of the system, which was defined by the ratio of the total counts on the CCD camera to the number of photon emitted from the Lanex to a hemisphere, was $(4.8 \pm 0.3) \times 10^{-6}$.

For the image processing, several effects were taken into account: (1) darkening at the edges of acquired images due to the finite collection solid angle, and (2) darkening/ brightening due to the $45^{\circ}$ orientation of the Lanex. The imaging system used the objectives instead of single lenses to realize a compact observation system [27], which made

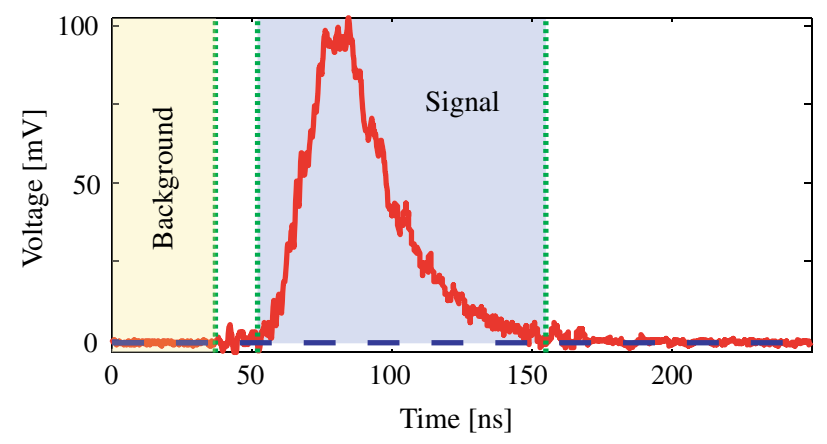

FIG. 3. Typical measured ICT signal (solid line). The background level was evaluated by averaging the signal between $0 \mathrm{~ns}$ and the first vertical dotted line, and is shown by the dashed line. To obtain charge, the signal between the second and the third vertical dotted line was integrated. theoretical modeling of the finite solid angle effect difficult. Therefore, that effect was experimentally measured.

In order to evaluate finite solid angle effects, the transverse and longitudinal light location dependences were measured off-line by using a green light emission diode (LED). A piece of Lanex was placed in front of the LED to imitate scattering from the Lanex, and position of the LED was scanned transversely to measure the darkening effect. Shown in Fig. 4(a) is the applied compensating factor for the image processing as a function of the number of pixels from the center pixel. The measurements were done with two different distances between the camera and the Lanex, confirming that the normalized edge darkening did not depend on the distance. One can see that about $10 \%$ darkening was observed at the edge.

To compensate distance dependence due to the $45^{\circ}$ orientation of the Lanex, the distance dependence was measured by longitudinally scanning the LED location. The compensating factor as a function of the Lanexcamera distance is shown in Fig. 4(b), where the factor was normalized to the distance from the Lanex at the center of the view to the camera $(213 \mathrm{~mm})$. The measured distance dependence was also used for the relative calibration between this setup and others with different distances [27].

Typical processed images for 1289 and $106 \mathrm{MeV}$ electron energy are shown in Figs. 4(c) and 4(d). As can be seen, the size of the electron beam on the Lanex depended on the energy of the electron. The compensation of the finite solid angle effect was essential for accurate measurements. The signal to noise ratio for each image was defined by $S N_{\text {img }}=C_{\text {peak }} / C_{\sigma \mathrm{BG}}$, where $C_{\text {peak }}$ is the peak counts of the image and $C_{\sigma \mathrm{BG}}$ is the standard deviation of the background image.

\section{B. Results}

Measurements of e-beam charge and light yield from the Lanex Fast Back were conducted for 15 different electron energies. An appropriate ND filter, which was calibrated with a spectrophotometer, was chosen for each measurement, so that the highest charge density could be explored. For each measurement, more than 100 shots were acquired scanning the gun bias voltage to explore a wide range of the total charge. Shown in Fig. 5(a) is a case for $889 \mathrm{MeV}$ electron energy with one microbunch. The horizontal axis is the charge measured by the ICT, and the vertical axis is the total counts on the CCD camera. Note that the large error seen for low charge $(<100 \mathrm{pC})$ points is not an intrinsic limitation of the Lanex measurement. It was due to the ND filter used and the dynamic range of the camera. The Lanex measurement can be set for much lower charge as shown in Sec. III.

From all the scans, offsets were observed as indicated in Fig. 5(a). Since these offsets were found to be systematic, and always negative for CCD camera counts, it was attributed to a threshold bias of the CCD camera electronics. 

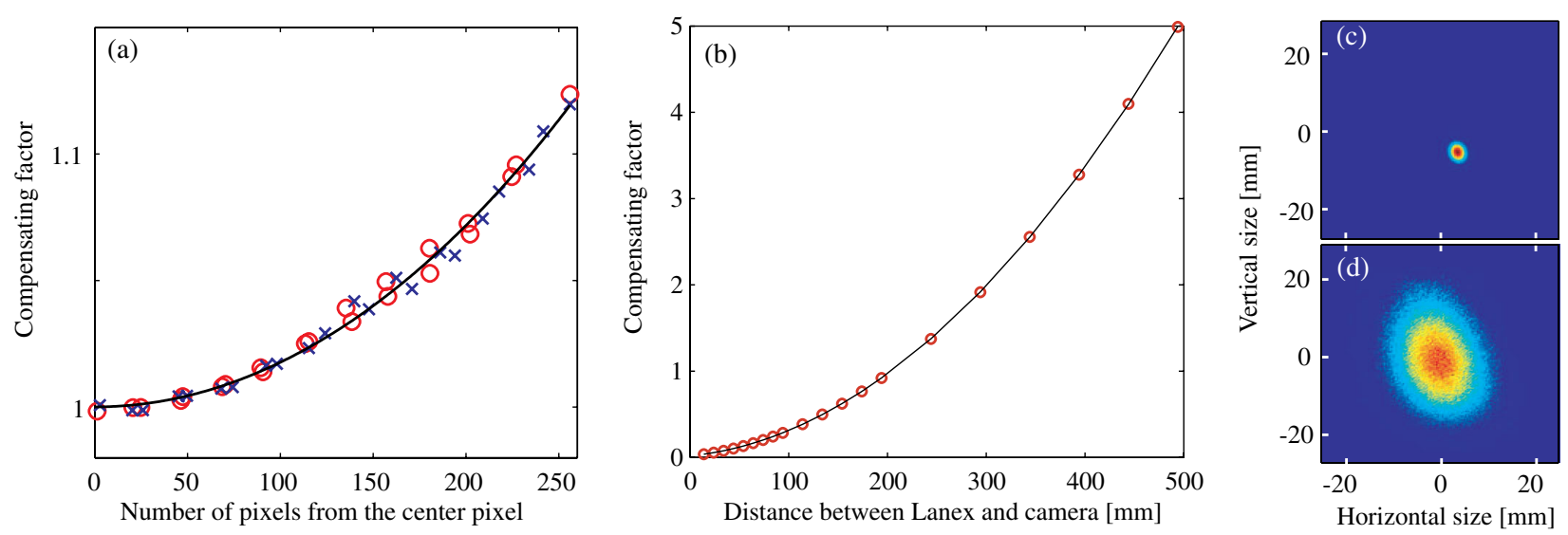

FIG. 4. (a) Measured compensating factor for the image processing based on the LED measurement for two different distances between the Lanex and the camera: circles, $100 \mathrm{~mm}$; crosses, $270 \mathrm{~mm}$. The horizontal axis is the number of pixels from the center pixel. Note that the compensating factor is normalized to the counts at the center pixel. The solid line is a second order polynomial fit result, which was used for the compensation. (b) Measured compensating factor as a function of the distance between the Lanex and camera (circles). The factor was normalized to the distance from the Lanex at the center of the view to the camera (213 mm). The solid line is a second order polynomial fit result, which was used for the compensation. (c),(d) Typical processed images for (c) $1289 \mathrm{MeV}$ and (d) $106 \mathrm{MeV}$ electron energy.
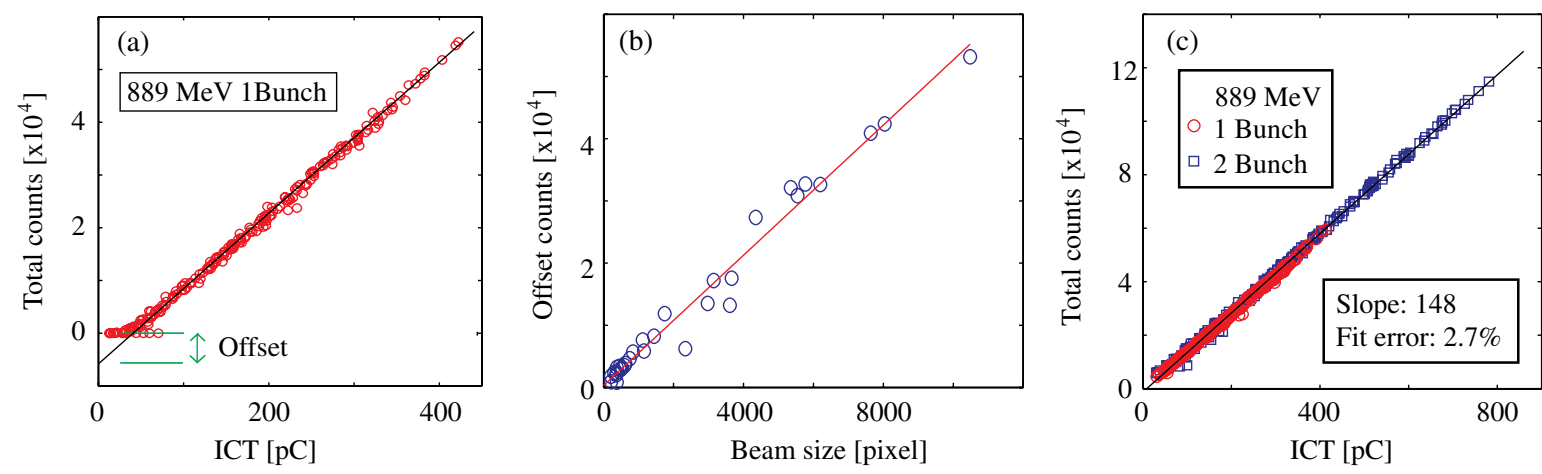

FIG. 5. (a) Typical charge scan results (circles). The horizontal axis is the charge measured by the ICT, and vertical axis is the total counts on the CCD camera. The electron energy was $889 \mathrm{MeV}$, and one microbunch was used. The solid line is a linear fit. The offset of the CCD counts is indicated by the arrow. (b) The observed offset CCD camera counts as a function of the beam size in terms of the number of pixels for 15 different energies with one and two microbunches (circles). The solid line is a linear fit. (c) The total counts on the CCD camera as a function of the charge measured by the ICT for $889 \mathrm{MeV}$ electron energy, circles: one microbunch; squares: two microbunches. The solid line is a linear fit, and the slope and the fit error defined by Eq. (1) are shown in the lower right inset. The total counts on the CCD camera were biased based on the modeled offset shown in (b), and only points with the signal to noise ratios for ICT $S N_{\text {ict }}$ and CCD camera image $S N_{\text {img }}$ above 10 were taken into account.

This threshold would give a negative offset proportional to the beam size. The observed offsets as a function of the beam size are shown in Fig. 5(b). The offsets and beam size were well correlated, which was consistent with a threshold-induced offset. In the analysis, the bias based on the modeled offset was applied for the total counts on the CCD camera. Note that this problem can be solved by employing a camera with higher sensitivity.

For each electron energy, measurements were done with one and two microbunch modes. Shown in Fig. 5(c) are the total counts on the CCD camera as a function of the measured charge by the ICT for $889 \mathrm{MeV}$ electron energy. The measurements of 1-bunch (circles) and 2-bunch (squares) modes are placed on top of each other. For this plot, the total counts on the CCD camera were biased based on the modeled offset shown in Fig. 5(b), and only points with the signal to noise ratios for ICT $S N_{\text {ict }}$ and CCD camera image $S N_{\text {img }}$ above 10 were taken into account. The linear fitting was done for the combined data set, and the slope is shown in the inset. To evaluate the quality of the fit, the fit error $\hat{\epsilon}$ is shown in the lower right box and is defined as the mean of the normalized standard deviation,

$$
\hat{\boldsymbol{\epsilon}}=\frac{1}{g} \sum^{g} \frac{\left|\mathrm{CCD}_{i}-f\left(\mathrm{ICT}_{i}\right)\right|}{f\left(\mathrm{ICT}_{i}\right)}
$$


where $g$ is the number of samples, $\mathrm{CCD}_{i}$ and $\mathrm{ICT}_{i}$ are the total counts on the CCD camera and measured charge by the ICT of the $i$ th sample, and $f\left(\mathrm{ICT}_{i}\right)$ is the total counts on the CCD camera predicted by the fitting result for the charge measured by the ICT. The fit errors were found to be from $1.0 \%$ to $5.6 \%$, with $2.8 \%$ for the average.

The electron energy dependence of the number of photons emitted from the Lanex is shown in Fig. 6. Based on the slopes for each electron energy and the total efficiency of the system described in Sec. II A, the number of photons emitted to a hemisphere per one electron was obtained. The errorbars show the convolution of the fit error $\hat{\epsilon}$ and the error of the total efficiency. The solid line is a linear fit for the Lanex Fast Back results, indicating that the energy dependence is well modeled by the linear fit. It was found that the Lanex Fast Back emits about 1\% less photons for every $100 \mathrm{MeV}$ increase of electron energy. The result of the Lanex Fast Front for $1522 \mathrm{MeV}$, and the results of Lanex Regular and Fine from Ref. [32] are shown for references. One can see that the Lanex Fast Back yielded about 2 times more light than the Lanex Fast Front, and about 6 times more light than the Lanex Fine.

The electron energy dependence of the Lanex light yield was not observed in previous works $[26,28,32]$. Since the previously explored energy range was small enough for the difference to be within the measurement error, the observed energy dependence is not contradictory to previous works. Although the material used was different, it is noteworthy that the IP showed similar energy dependence $[29,30]$, where the sensitivity of IP decreased $\sim 4 \%$ per $100 \mathrm{MeV}$ increase of the energy.

There are a few possible scenarios that may explain the observed energy dependence. Relativistic electrons ionize

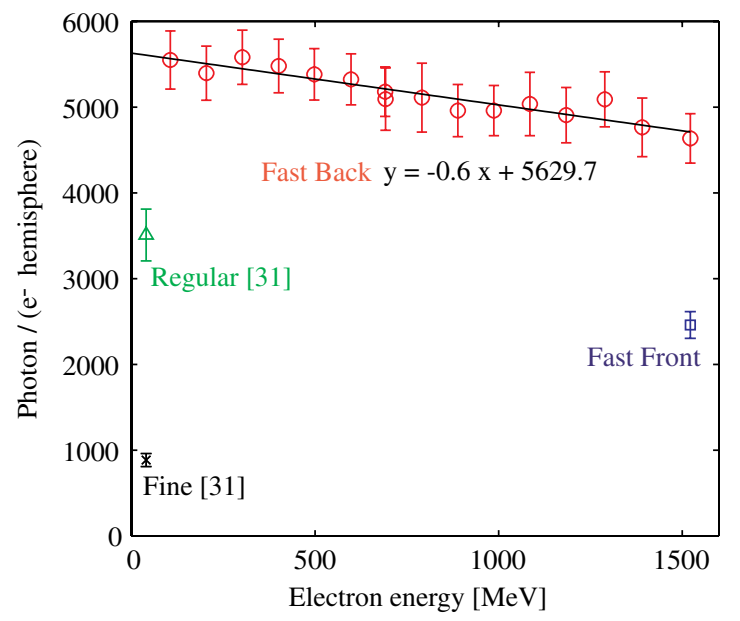

FIG. 6. The number of photons emitted from the Lanex per electron as a function of the electron energy: circles, Lanex Fast Back; square, Lanex Fast Front; triangle, Lanex Regular [32]; cross, Lanex Fine [32]. The solid line is a linear fit for the Lanex Fast Back. The errorbars are the convolution of the fit error and the error of the total efficiency. material, exciting a certain level followed by the radiative relaxation, and also produce bremsstrahlung $\gamma$ rays, that can create knock-on electrons which also ionize the material. Since higher energy photons get absorbed less in the material [39], the contribution from bremsstrahlung $\gamma$ rays may become less for higher energy electrons. Another possible scenario is that the electron scattering angle is less for higher energy electrons, resulting in less interaction with the material. Since the observed energy dependence was fairly small, the effect from the different beam size on the screen cannot be ruled out for the cause of this. Although all the geometrical effects were taken into account such as the edge darkening and the distance dependence, there still could be small effects that were not modeled in the analysis.

For all the scans described above, linear relationships were observed between the Lanex light yield and the ICT measured charge. The charge density was explored up to $160 \mathrm{pC} / \mathrm{mm}^{2}$ in $\sim 8 \mathrm{~ns}$ for $1391 \mathrm{MeV}$ electron beam with two microbunch mode. With one bunch mode, the charge intensity was explored up to $0.4 \mathrm{pC} / \mathrm{ps} / \mathrm{mm}^{2}$. The linear response of the Lanex Fast Back was verified up to those parameters.

\section{CHARGE DIAGNOSTICS CROSS CALIBRATIONS WITH LASER PLASMA ACCELERATOR}

In this section, a study of three charge diagnostics using LPA produced e-beams is presented. The activation-based measurement [23] is intrinsically immune to EMP noise, and electron charge intensity independent. Therefore, it can provide reliable reference for LPA produced e-beam charge measurements. The Lanex has been calibrated using RFAs [26,32], but has not been benchmarked with a reliable diagnostic for LPA produced e-beams. The use of an ICT has been questioned for LPA produced e-beams charge measurement, while it has been used as a reliable reference for RFAs. The experimental setup is shown in Sec. III A, the Lanex-activation cross calibration in Sec. III B, and the Lanex-ICT cross calibration in Sec. III C. It is shown that all the diagnostics can provide accurate charge measurements for LPA produced e-beam.

\section{A. Experimental setup}

Cross calibrations between the Lanex and activationbased method, and between the Lanex and ICT, were conducted by using LPA produced e-beams at the LOASIS facility, LBNL [40]. The laser that was utilized was a short pulse, high peak power and high repetition rate $(10 \mathrm{~Hz}) \mathrm{Ti}: \mathrm{Al}_{2} \mathrm{O}_{3}$ laser system. The laser beam was focused by an off-axis parabolic mirror, providing a focal spot size $r_{0} \simeq 23 \mu \mathrm{m}$ with Strehl ratio of 0.9. Here, a Gaussian transverse profile of $I=I_{0} \exp \left(-2 r^{2} / r_{0}^{2}\right)$ is assumed. Full energy and optimum compression gives $P=$ $31 \mathrm{TW}\left(\tau_{\text {in }} \simeq 40 \mathrm{fs}\right.$ FWHM $)$, calculated peak intensity 


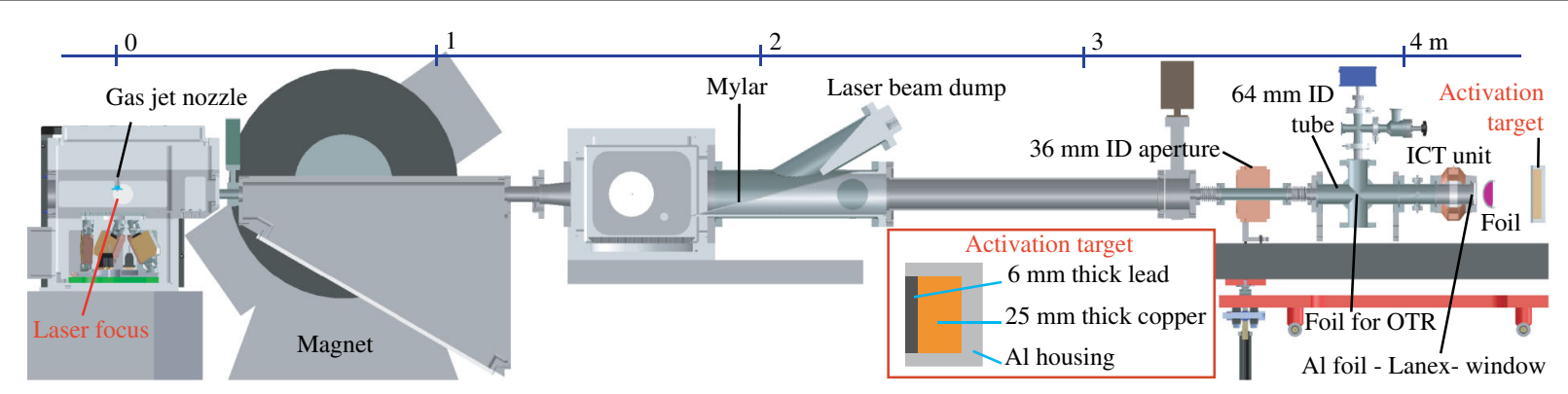

FIG. 7. Setup for the charge cross calibrations with LPA produced e-beams. The laser pulse was focused onto a supersonic gas jet. The laser pulse was reflected toward a laser beam dump by an aluminum coated Mylar foil. An aperture with a $36 \mathrm{~mm}$ inner diameter was installed about $3.6 \mathrm{~m}$ away from the interaction point. An aluminum coated $5 \mu \mathrm{m}$ thick pellicle foil was located about $3.9 \mathrm{~m}$ for an optical transition radiation experiments (OTR foil). The identical ICT unit described in Fig. 2 followed by the borosilicate vacuum window, was installed at the end of the vacuum tube. The Lanex Fast Front was placed on the window inside of the vacuum tube, upstream of which was covered by a $\simeq 40 \mu \mathrm{m}$ thick aluminum foil. The light from the Lanex was observed by a CCD camera (not shown) through the reflection of an aluminum coated $5 \mu \mathrm{m}$ thick pellicle foil places with a 45 -degree angle. Shown in the inset is the activation target that consisted of the $6 \mathrm{~mm}$ thick lead and the $25 \mathrm{~mm}$ thick copper in the aluminum housing.

$I_{0}=2 P / \pi r_{0}^{2} \simeq 3.4 \times 10^{18} \mathrm{~W} / \mathrm{cm}^{2}$, and a normalized vector potential $a_{0} \simeq 8.6 \times 10^{-10} \lambda[\mathrm{m}] I^{1 / 2}\left[\mathrm{~W} / \mathrm{cm}^{2}\right] \simeq 1.3$.

The schematic drawing of the setup is shown in Fig. 7 with a scale to indicate the distance from the interaction point. The laser pulse came from the left, and was focused onto a supersonic gas jet. A magnet for electron spectrometer was located about $0.7 \mathrm{~m}$ downstream of the interaction point. For the cross calibrations, the magnet was turned off to send e-beams to charge diagnostics located further downstream. The laser pulse was reflected by the aluminum coated Mylar foil toward the laser beam dump, and only e-beams went through following vacuum tubes.

An aperture with a $36 \mathrm{~mm}$ inner diameter was installed about $3.6 \mathrm{~m}$ away from the interaction point. An aluminum coated $5 \mu \mathrm{m}$ thick pellicle foil was located about $3.9 \mathrm{~m}$ for an optical transition radiation experiments (OTR foil). The identical ICT unit described in Fig. 2 followed by the borosilicate vacuum window, was installed at the end of the vacuum tube. The Lanex Fast Front (64 mm diameter) was placed on the window, inside of the vacuum tube, upstream and was covered by $\mathrm{a} \simeq 40 \mu \mathrm{m}$ thick aluminum foil (see Sec. II A). There was no loss of e-beam between the ICT and the Lanex, because the angle acceptance of the ICT and the Lanex was larger than the acceptances of the aperture and the OTR foil.

The light from Lanex was observed by an identical CCD camera described in Sec. II A (not shown in Fig. 7) through the reflection of an aluminum coated $5 \mu \mathrm{m}$ thick pellicle foil installed in the outside of the vacuum tube. The reflectivity of the foil was measured to be $(0.97 \pm$ 0.02). For the activation measurement, the target was placed behind the pellicle foil. The target consisted of $6 \mathrm{~mm}$ thick lead as a $\gamma$-ray generator followed by the $25 \mathrm{~mm}$ thick copper as an activation material, and is illustrated in the Fig. 7 inset.

\section{B. Lanex-activation cross calibration}

The activation-based charge diagnostic was used as follows. The target material was irradiated by e-beams for $\simeq 1 \mathrm{~h}$. After the irradiation, the target was transferred to an ultralow background counting facility at LBNL, where $\gamma$-ray spectroscopy was conducted. Based on the $\gamma$-ray spectroscopy results, the activity in terms of the average production rate $R_{\text {exp }}$ (atoms/minute) for a specific isotope was calculated. In order to estimate the charge, a Monte Carlo simulation was carried out to calculate the yield of an isotope for a unit charge with a certain e-beam energy spectrum $Y_{\text {sim }}$ (atoms/electron). The e-beam energy spectrum was separately measured during the experiments. The number of electrons per minute irradiated to the target $\bar{N}_{e}$ was obtained from $\bar{N}_{e \text { Act }}=R_{\exp } / Y_{\text {sim }}$. As the result, the activation method can provide the time-averaged charge for e-beams with known energy spectrum. The detailed description of the method is provided in Ref. [23].

The laser pulse was focused onto the hydrogen jet to produce high charge relativistic e-beams. The plasma density was measured by transverse interferometry. The peak plasma density was measured to be $\sim 1.9 \times 10^{19} / \mathrm{cm}^{3}$, and plasma longitudinal length was $\sim 0.9 \mathrm{~mm}$. The e-beam energy spectra were measured by a single-shot magnetic electron spectrometer [27] before sending the e-beam to charge diagnostics, and reproducible broadband e-beams up to $250 \mathrm{MeV}$ were observed. The reference e-beam spectrum, which is the average of 50 shots, is shown in Fig. 8(a). The solid line shows the average spectrum, and the grey area shows the \pm standard deviation. The normalized standard deviation of the total charge was found to be $32 \%$.

The e-beams were sent to the activation target by turning off the magnet of the electron spectrometer. A total of 2700 shots was incident onto the target in 60 minutes. The aluminum coated $5 \mu \mathrm{m}$ thick pellicle foil was placed 


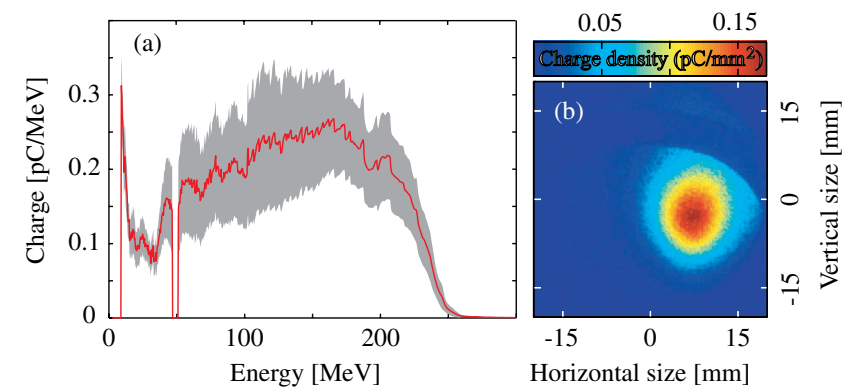

FIG. 8. (a) Reference e-beam spectrum for the activation measurement. The solid line shows the average spectrum, and the grey area shows the \pm standard deviation. A total of 50 shots was taken. The normalized standard deviation of the total charge was found to be $32 \%$. (b) Averaged e-beam profile measured by the Lanex during the activation. A total of 2700 shots was acquired, and the average charge was $37 \mathrm{pC} /$ shot. The horizontal beam size in FWHM was measured to be $11.5 \mathrm{~mm}$, and the vertical size was $13.7 \mathrm{~mm}$.

between the Lanex and the activation target to separate the light from the Lanex and e-beams with minimal disturbance.

During the activation, the e-beam charge was simultaneously measured by the Lanex. The average e-beam profile is shown in Fig. 8(b). The e-beam charge was measured to be $(36.6 \pm 1.3) \mathrm{pC} / \mathrm{shot}$, or the number of electrons in a minute measured by the Lanex $\bar{N}_{e \operatorname{Lnx}}=(10.3 \pm 0.4) \times$ $10^{9}$ electrons/minute. The error of the Lanex charge measurement was $3.4 \%$, obtained from the convolution of the average fit error described in Sec. II B and the error in the reflectivity of the foil. The horizontal beam size in FWHM was measured to be $11.5 \mathrm{~mm}$, and the vertical size was $13.7 \mathrm{~mm}$. The sharp edge on the top right was due to the retaining ring of the OTR foil (see Fig. 7 for location).

After the irradiation, the target was transferred to the counting facility, and $\gamma$-ray spectroscopy was conducted by using a $p$-type HPGe detector. The $1345 \mathrm{keV}$ photons from ${ }^{64} \mathrm{Cu}$ decay (half lifetime $12.7 \mathrm{~h}$ ) was used to determine the yield of the isotope. Assuming constant production rate during the irradiation, the average production rate $R_{\exp }$ was measured to be $(9.79 \pm 0.59) \times 10^{6}$ atoms/minute, where the error was given by the $\pm 1 \sigma$ (standard deviation).

A Monte Carlo simulation was carried out to estimate isotope production based on the e-beam reference spectrum using MCNPX code [41] and NNDC database [42]. An axisymmetric, three-dimensional distribution of the isotope production was calculated. The incident angle of electrons were assumed to be $0^{\circ}$, and the transverse profile of the e-beam was assumed to be a Gaussian shape with $13 \mathrm{~mm}$ FWHM based on the Lanex measurements. In the simulation, energy loss of electrons due to the interactions with the foil, Lanex, vacuum window, and air were included. Based on the 3D activation distribution, self-attenuation of emitted $\gamma$ ray was taken into account. The simulated yield of the isotope $Y_{\text {sim }}$ was
$1.02 \times 10^{-3}$ atoms/electron, giving $\bar{N}_{e \text { Act }}=R_{\exp } / Y_{\text {sim }}=$ $(9.6 \pm 0.6) \times 10^{9}$ electrons/minute.

The charge measured by the activation-based measurement $\bar{N}_{e \text { Act }}=(9.6 \pm 0.6) \times 10^{9}$ and the Lanex measurement $\bar{N}_{\text {eLnx }}=(10.3 \pm 0.4) \times 10^{9}$ agreed with each other within the error of each measurement. This was the first time that the charge diagnostic based on a phosphor screen was benchmarked against a reliable method for LPA produced e-beam, and indicated that the Lanex can be an accurate charge diagnostic for LPA produced e-beams.

\section{Lanex-ICT cross calibration}

In order to produce relativistic e-beams, the laser pulses were focused onto the downstream edge of a gas jet comprised of $99 \%$ helium and $1 \%$ nitrogen. The gas jet backing pressure was varied to change charge yield from the LPA. The peak plasma density was measured by transverse interferometry, and was found to be from 4 to $10 \times 10^{18} / \mathrm{cm}^{3}$, and the longitudinal plasma length was about $0.5 \mathrm{~mm}$. Reproducible e-beams up to $60 \mathrm{MeV}$ were observed, and the e-beam spectra as a function of the peak plasma density are shown in Fig. 9. Higher plasma density resulted in producing e-beams with the higher charge and the broader spectrum.

The Lanex-ICT cross calibration was carried out in the same manner as the calibration with RFA-generated e-beams. Electron beams were sent to the various charge diagnostics by turning off the magnet for the spectrometer. A total of 320 shots was recorded while varying the plasma density, and e-beams with the charge up to $16 \mathrm{pC}$ were observed. Shown in Fig. 10 are (a) a typical measured e-beam profile on the Lanex, (b) a typical measured ICT signal, and (c) the charge measured by the Lanex versus the charge measured by the ICT.

The sharp edge of the e-beam profile was due to the $36 \mathrm{~mm}$ ID aperture at $3.7 \mathrm{~m}$ and the holder for the retaining

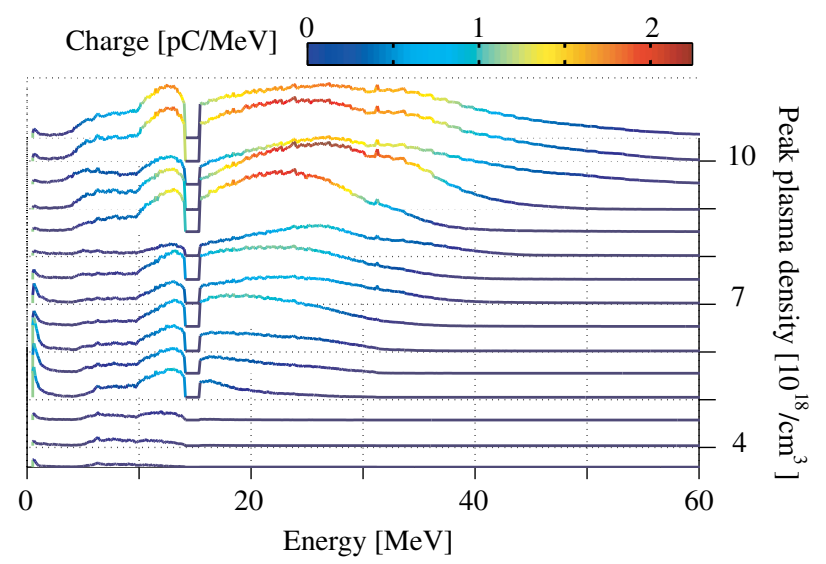

FIG. 9. Electron beam spectra as a function of the peak plasma density. Each spectrum is an average of eight shots. The dip at around $17 \mathrm{MeV}$ is due to the gap in the electron spectrometer [27]. 

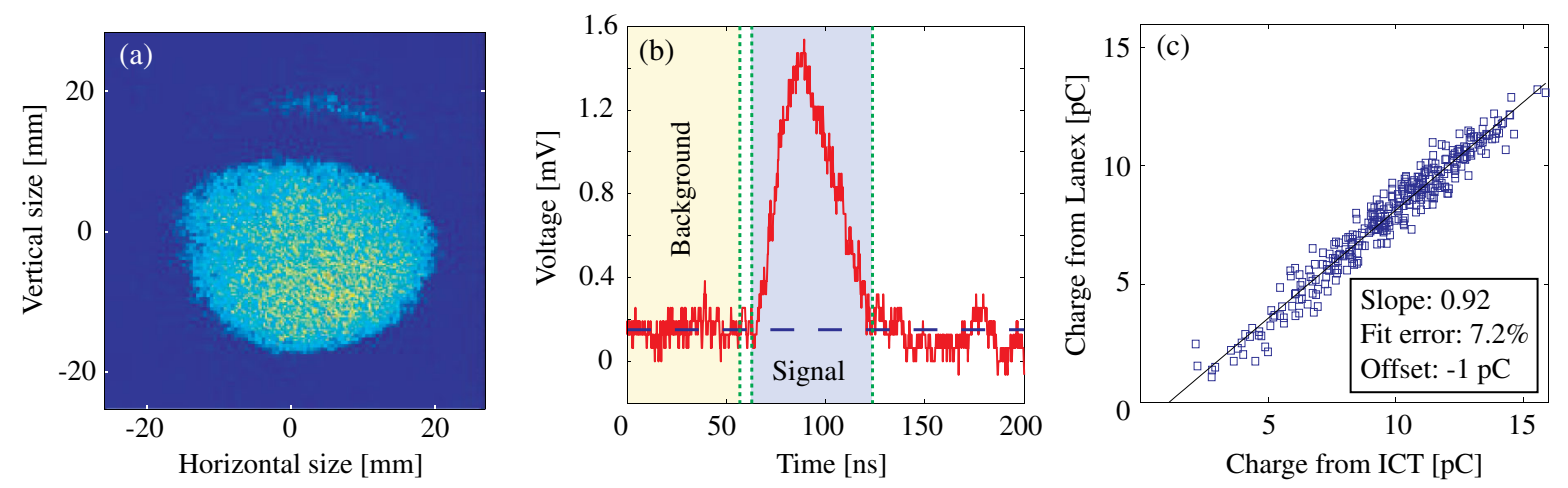

FIG. 10. (a) Typical measured e-beam profile with the Lanex. (b) Typical measured ICT signal (solid line). (c) Lanex measured charge as versus ICT measured charge. The solid line is a linear fit, and the slope and offset are shown in the inset as well as the fit error. The total counts on the CCD camera were biased based on the analysis in Sec. II B, and points with both $S N_{\text {ict }}$ and $S N_{\text {img }}$ above 10 were taken into account.

ring of the OTR foil. The image and waveform analysis was performed in the same way described in Sec. II A. For Fig. 10(c), the total counts on the CCD camera were biased based on the analysis in Sec. II B, and points with both $S N_{\text {ict }}$ and $S N_{\text {img }}$ above 10 were taken into account. A linear fit performed on the data set indicated that the Lanex measured $8 \%$ lower charge with the offset of $-1 \mathrm{pC}$. The two diagnostics showed good agreement considering the fit error of $7.2 \%$. The $1 \mathrm{pC}$ of negative offset was still observed after the compensation. It was attributed to the difference of the transverse profiles of RFA and LPA e-beams. The tail of the e-beam, where the camera cannot see due to the sensitivity, may differ. As discussed in Sec. II B, this problem can be solved by employing a camera with higher sensitivity.

As shown above, the ICT can measure the LPA produced e-beam charge accurately, while previous works showed that the ICT overestimated the charge up to 2 orders of magnitude. The excellent agreement we observed can be explained by the special attention paid to the following three possible noise sources of the ICT measurement: (1) EMP from the laser-plasma interaction, (2) direct particle/ radiation hit on the ICT, and (3) low energy electrons.

There were two kinds of EMP noises observed, one was directly on the scope, and the other was on the cable and/or the ICT. The noise on the scope was separated from the signal in the time domain by extending the cable length. To minimize the noise on the cable, well-shielded cables (Heliax FSJ1-50A, CommScope, Hickory, NC, United States) were employed, and the route of the cables was carefully arranged to reduce the noise. Since the higher frequency part of the EMP noise was visible from the waveform, it was used as an indicator while optimizing the route. As can be seen from Fig. 10(b), the obtained ICT signals did not contain high frequency spikes.

The direct hit of the laser pulse or e-beam onto the ICT can potentially create secondary electrons and/or ionize the material, possibly contributing to the noise. The laser pulse was separated from e-beams to prevent it from hitting the
ICT. An aperture was utilized for e-beam transverse size to be smaller than the acceptances of the ICT and Lanex, assuring that the e-beams did not hit the ICT or vacuum tube. The ICT was installed outside of the vacuum tube over a ceramic gap so that e-beams propagate in vacuum with minimum disturbance.

The low energy electrons could cause a large discrepancy between the ICT and Lanex measurements because of the following reasons. (1) Nonlinear beam size evolution due to the space charge effect can lead to the acceptance mismatch between the two diagnostics. (2) The response of the Lanex against low energy electron $(<1 \mathrm{MeV})$ is not clear. In this experiment, the ICT was installed $4.2 \mathrm{~m}$ away from the interaction point to assure that the low energy electrons diverge enough to minimize their contribution. Furthermore, the small residual magnetic field $(<0.4 \mathrm{mT})$ of the magnetic spectrometer, and absorptions/scatterings at the foils used for the laser beam separation may have contributed to eliminate low energy electrons. The distance between ICT and Lanex was kept at a minimum to avoid acceptance mismatch.

Although no quantitative evaluation was performed for each noise source, it was considered to be critical to provide a low-noise environment for the charge measurement. Note that the accuracy of the measurement can be improved by a more sensitive camera for Lanex measurements, and by more sensitive electronics for ICT measurements.

\section{SUMMARY AND CONCLUSIONS}

The electron energy dependence of the Lanex Fast light yield was studied from 106 to $1522 \mathrm{MeV}$ e-beams. The Lanex was observed to be $1 \%$ less sensitive for every $100 \mathrm{MeV}$ increase in the energy. The charge density and the charge intensity on the Lanex was explored up to $160 \mathrm{pC} / \mathrm{mm}^{2}$ in $\sim 8 \mathrm{~ns}$, and $0.4 \mathrm{pC} / \mathrm{ps} / \mathrm{mm}^{2}$, respectively. The linear response of the Lanex Fast Back was verified up to those parameters. 
A comprehensive study of the charge diagnostics for LPA produced e-beams was conducted. The cross calibration between the Lanex and the activation-based charge diagnostic showed good agreement within the error of each diagnostic. This was the first benchmark of the Lanex against a reliable method for LPA produced e-beam charge measurement. The cross calibration between the Lanex and the ICT showed good agreement as well. The result of the cross calibrations can be summarized as follows: $Q_{\text {Act }}=$ $93_{87}^{98}, Q_{\mathrm{Lnx}}=100_{97}^{103}, Q_{\mathrm{ICT}}=108_{100}^{116}$, where they are normalized to the Lanex result, and superscripts and subscripts show result \pm the error of each diagnostics. The error for the ICT measurement is taken from the fit error of the Lanex-ICT cross calibration using LPA produced e-beams. This study showed that all diagnostics can provide accurate charge measurement for LPA produced e-beams. The guideline for accurate measurements with the ICT under the harsh LPA environment was discussed. This study will provide essential information for charge measurements of LPA produced e-beams.

\section{ACKNOWLEDGMENTS}

The authors acknowledge all the ALS staff, Bas Fleskens, Pablo Gallegos, and Eamonn Monaghan for contribution on the experiments at the ALS, Dmitriy Panasenko, Thomas Sokollik, Jens Osterhoff, Satomi Shiraishi, Jeroen van Tilborg, and Csaba Toth for their contributions on the LPA experiments, and Don Syversrud, Nathan Ybarrolaza, Zachary Eisentraut, and Adam Brown for technical support. We also thank Julien Bergoz for the fruitful discussion on integrating current transformers, and Cameron Geddes, Nicholas Matlis, Guillaume Plateau, Carl Schroeder, and Eric Esarey for their contributions. This work was supported by the U.S. Department of Energy under Contract No. DE-AC02-05CH11231.

[1] E. Esarey, C. B. Schroeder, and W. P. Leemans, Rev. Mod. Phys. 81, 1229 (2009).

[2] C. G. R. Geddes, C. Tóth, J. van Tilborg, E. Esarey, C. B. Schroeder, D. Bruhwiler, C. Nieter, J. Cary, and W. P. Leemans, Nature (London) 431, 538 (2004).

[3] S. Mangles et al., Nature (London) 431, 535 (2004).

[4] J. Faure, Y. Glinec, A. Pukhov, S. Kiselev, S. Gordienko, E. Lefebvre, J.-P. Rousseau, F. Burgy, and V. Malka, Nature (London) 431, 541 (2004).

[5] W. P. Leemans, B. Nagler, A. J. Gonsalves, C. Tóth, K. Nakamura, C. G. R. Geddes, E. Esarey, C. B. Schroeder, and S. M. Hooker, Nature Phys. 2, 696 (2006).

[6] K. Nakamura, B. Nagler, C. Tóth, C. G. R. Geddes, C. B. Schroeder, E. Esarey, W. P. Leemans, A. J. Gonsalves, and S. M. Hooker, Phys. Plasmas 14, 056708 (2007).

[7] A. J. Gonsalves, T.P. Rowlands-Rees, B. H. P. Broks, J. J. A. M. van der Mullen, and S. M. Hooker, Phys. Rev. Lett. 98, 025002 (2007).
[8] E. Esarey, R. F. Hubbard, W. P. Leemans, A. Ting, and P. Sprangle, Phys. Rev. Lett. 79, 2682 (1997).

[9] S. Bulanov, N. Naumova, F. Pegoraro, and J. Sakai, Phys. Rev. E 58, R5257 (1998).

[10] E. Oz et al., Phys. Rev. Lett. 98, 084801 (2007).

[11] C. G. R. Geddes, K. Nakamura, G. R. Plateau, C. Toth, E. Cormier-Michel, E. Esarey, C. B. Schroeder, J. R. Cary, and W. P. Leemans, Phys. Rev. Lett. 100, 215004 (2008).

[12] J. Faure, C. Rechatin, A. Norlin, A. Lifschitz, Y. Glinec, and V. Malka, Nature (London) 444, 737 (2006).

[13] A. J. Gonsalves, E. Esarey, C. G. R. Geddes, W.P. Leemans, C. Lin, K. Nakamura, D. Panasenko, C. B. Schroeder, and C. Toth, in Proceedings of the 2009 Particle Accelerator Conference (IEEE, Piscataway, NJ, 2009).

[14] A. Pak, K. A. Marsh, S. F. Martins, W. Lu, W. B. Mori, and C. Joshi, Phys. Rev. Lett. 104, 025003 (2010).

[15] C. McGuffey et al., Phys. Rev. Lett. 104, 025004 (2010).

[16] W.P. Leemans et al., Phys. Rev. Lett. 91, 074802 (2003).

[17] S. Kneip et al., Phys. Rev. Lett. 100, 105006 (2008).

[18] M. Fuchs et al., Nature Phys. 5, 826 (2009).

[19] K. B. Unser, in Proceedings of the 1989 Particle Accelerator Conference (IEEE, Piscataway, NJ, 1989), Vol. 1, p. 71.

[20] C. E. Clayton, M. J. Everett, A. Lal, D. Gordon, K. A. Marsh, and C. Joshi, Phys. Plasmas 1, 1753 (1994).

[21] D. Umstadter, S.-Y. Chen, A. Maksimchuk, G. Mourou, and R. Wagner, Science 273, 472 (1996).

[22] C. Gahn, G. D. Tsakiris, K. J. Witte, P. Thirolf, and D. Habs, Rev. Sci. Instrum. 71, 1642 (2000).

[23] W. P. Leemans, D. Rodgers, P. E. Catravas, C. G. R. Geddes, G. Fubiani, E. Esarey, B. A. Shadwick, R. Donahue, and A. Smith, Phys. Plasmas 8, 2510 (2001).

[24] L. M. Chen, J. J. Park, K.-H. Hong, J. L. Kim, J. Zhang, and C. H. Nam, Phys. Rev. E 66, 025402 (2002).

[25] M. Galimberti, A. Giulietti, D. Giulietti, and L. A. Gizzi, Rev. Sci. Instrum. 76, 053303 (2005).

[26] Y. Glinec, J. Faure, A. Guemnie-Tafo, V. Malka, H. Monard, J.P. Larbre, V.D. Waele, J.L. Marignier, and M. Mostafavi, Rev. Sci. Instrum. 77, 103301 (2006).

[27] K. Nakamura, W. Wang, N. Ybarrolaza, D. Syversrud, J. Wallig, and W. Leemans, Rev. Sci. Instrum. 79, 053301 (2008).

[28] S. Masuda, E. Miura, K. Koyama, and S. Kato, Rev. Sci. Instrum. 79, 083301 (2008).

[29] K. A. Tanaka, T. Yabuuchi, T. Sato, R. Kodama, Y. Kitagawa, T. Takahashi, T. Ikeda, Y. Honda, and S. Okuda, Rev. Sci. Instrum. 76, 013507 (2005).

[30] N. Nakanii et al., Rev. Sci. Instrum. 79, 066102 (2008).

[31] K. Zeil, S. D. Kraft, A. Jochmann, F. Kroll, W. Jahr, U. Schramm, L. Karsch, J. Pawelke, B. Hidding, and G. Pretzler, Rev. Sci. Instrum. 81, 013307 (2010).

[32] A. Buck et al., Rev. Sci. Instrum. 81, 033301 (2010).

[33] N. A. M. Hafz et al., Nat. Photon. 2, 571 (2008).

[34] C. E. Clayton et al., Phys. Rev. Lett. 105, 105003 (2010).

[35] B. Hidding et al., Rev. Sci. Instrum. 78, 083301 (2007).

[36] W. Leemans, G. Behrsing, K.-J. Kim, J. Krupnick, C. Matuk, F. Selph, and S. Chattopadhyay, in Proceedings of the 1993 Particle Accelerator Conference (IEEE, Piscataway, NJ, 1993), pp. 83-85. 
[37] Technical Report No. PUB-5172 Rev., Lawrence Berkeley National Laboratory, University of California, Berkeley, 1986.

[38] PointGrey (private communication).

[39] Technical Report No. AN-07, Rad-Icon Imaging Corp., Santa Clara, CA, 2002, http://www.rad-icon .com.
[40] LOASIS facility, http://loasis.lbl.gov.

[41] D. B. Pelowitz, M.R. James, G. W. McKinney, J. W. Durkee, M. L. Fensin, J.S. Hendricks, S. G. Mashnik, and L.S. Waters, Technical Report No. LA-UR-0904150, Los Alamos National Lab, 2009.

[42] NNDC CSISRS database, http://www.nndc.bnl.gov/exfor/ exfor00.htm. 